\title{
Experience with Restoration of Asia Pacific Network Failures from Taiwan Earthquake
}

\author{
Yasuichi Kitamura ${ }^{1}$, Youngseok Lee ${ }^{2}$, \\ Ryo Sakiyama ${ }^{3}$ and Koji Okamura ${ }^{3}$ \\ ${ }^{1}$ National Institute of Information and Communications Technology, \\ ${ }^{2}$ Chungnam National Univeristy, \\ ${ }^{3}$ Kyushu University \\ 1,3 Japan \\ ${ }^{2}$ Korea
}

\section{Introduction}

As the Internet grows, networks become larger and more complex, and the number of components, such as routers, switches, and fiber cables, increases. In complicated network systems, it is difficult to implement global network management across several Internet service providers (ISPs) that use a lot of network components in a large-scale network topology. Fault management is a particularly important network management issue in complex network systems because the Internet has become essential to business and research. However, we are only beginning to learn how to deal with global network failures in large networks.

Failures have been reported in Sprint Internet protocol (IP) backbone, which shows that failures can be observed in everyday operation (Iannanccone et al., 2002; Markopulou et al., 2004). However, the network failures observed by (Iannaccone et al., 2002) and (Markopoulou et al., 2004) were short-lived and small scale, and their impacts were analyzed only in the context of a single ISP. Most network backup or fault restoration methods have been studied and proposed for the various layers such as wavelength division multiplexing (WDM), multi-protocol label switching (MPLS), or IP (Fumagalli \& Valcarenghi, 2000; Gerstel \& Ramaswami, 2000; Ramamurthy et al, 2003; Saharabuddhe et al., 2004; Sharma \& Hellstrand, 2003). Yet, the proposed backup and restoration methods have not been fully implemented and deployed in the real network. Since real networks are more complicated than theoretical ones, the impacts of network failures on users and ISP's cannot be completely predicted and analyzed. Significant network failures due to natural disasters such as earthquakes, floods, or fires could have particularly wide impact on several ISPs.

We discuss the results of the critical network failures that occurred after the Taiwan earthquake in Dec. 2006, which cut fibers and caused network failures. We also explain how restoration methods such as automatic border gateway protocol (BGP) (Lougheed \& Rekhter, 1989) re-routing, BGP policy change, and switch reconfiguration were conducted. We hope that the experience and knowledge we gained during the process of recovering 
from this huge natural disaster, which affected the global Internet, can be shared and can contribute to future Internet network management research. To the best of our knowledge, this is the first detailed study of network restoration after global network failures due to a natural disaster.

Although many natural disasters have occurred in the 21st century, until recently there had been no simultaneous outage of the global Internet backbone. However, the earthquake that occurred around Taiwan in 2006 made several Asia Pacific Research and Education (R\&E) networks unreachable. At 21:26 and 21:34 on December 26th (UTC+9), 2006, there was a big undersea earthquake off the coast of Taiwan twice, which measured 7.1 and 6.9 respectively on the moment magnitude (Hanks \& Kanamori, 1979). This earthquake caused significant damage to the undersea fiber cable systems in that area. Several ISPs were affected because each cable system is shared by multiple ISP's. This earthquake had the effect of dividing the Asia Pacific R\&E networks into an eastern and a western group. The Asia Pacific R\&E networks were, in particular, seriously damaged but were fully restored after several restoration steps, including automatic BGP re-routing, BGP policy changes, and switch port reconfigurations, were taken.

The first step in recovery after the earthquake was taken automatically by BGP routers, which detoured traffic along redundant routes. In BGP routing, there are usually multiple redundant AS paths. Redundant BGP routes have served as backup paths but have provided poor quality connectivity, i.e., long round trip time (RTT). Because of the congestion on the narrow-bandwidth link that was subsequently reported, operators took manual control of traffic to improve communication quality. The second step was a traffic engineering process intended to prevent narrow-bandwidth links from filling up with detoured traffic. The operators changed the BGP routing policy related to the congested ASs. In spite of the routing-level restoration, a few institutions were still not directly connected to the R\&E network community because they had only a single link to the network. For these single-link networks, the commodity link was used temporarily for connectivity. However, the commodity link was not stable and not sufficient to carry a huge amount of bandwidth or to provide next generation Internet service. To restore the singlelink networks, cable connection configurations at the switches were changed.

The fiber break caused by the Taiwan earthquake raised restoration issues related to BGP rerouting. In such an emergency, the backup routes should be chosen based on available bandwidth and RTT. Since the fiber break required an urgent network recovery process, network operators configured re-routing based on their experience with bandwidth and RTT.

From this experience, we have learned that redundant physical backup links and routes are important to providing bandwidth and connectivity and that the Quality-of-Service (QoS) after recovery is also important. From the viewpoint of restoration after network failures, there are still challenges that cannot be automatically overcome by network management systems. A systematic risk management plan that includes collaboration among operators of the next-generation Internet is needed.

The remainder of this chapter is as follows. In Section 2, the Asia Pacific R\&E networks that were damaged by the earthquake or related events are introduced. Section 3 introduces the R\&E connection especially in Asia Paicifc area and the issues caused by such inter-connectivity of R\&E networks. Section 4 is a detailed report of the network failures that were observed after the earthquake. Section 5 describes the processes to restoring the disrupted communications in the area. Section 6 discusses what we have learned from the observation of the network failures and recovery processes. Finally, we conclude the paper in Section 7. 


\section{Research and education network activities in the Asia Pacific area}

\subsection{Asian Internet Interconnection Initiative (AlII)}

When AIII (Asian Internet Interconnection Initiative [AIII], n.d.) started in 1996, this project was the first next generation Internet R\&E network in Asia. The basic idea of AIII is to provide Internet services via satellite. There are AIII members in TH, MY, ID, and SG (Tbl. 3). NP also recently joined the project. The AIII's major effort is to build up the access points of non-broadband networks. In Asia, it was very hard to complete the network over telephones lines, and preparing earth stations represented a better chance of accessing the Internet. The biggest drawback of this project is that the total bandwidth is limited to between 1.5 and 8 Mbps. In 1996, 2 Mbps was enough bandwidth to start research activities. Now, however, even $8 \mathrm{Mbps}$ is insufficient for network technology research activities. These days, AIII concentrates on developing and deploying certain advanced technology on their network, for example, IPv6 unicast, IPv6 multicast, Uni Directional Link Routing (UDLR), and advanced TCP.

\subsection{Asia-Pacific Advanced Network (APAN)}

APAN (Asia-Pacific Advanced Network [APAN], n.d.) started in 1997. APAN is the research consortium as well as it operates a next generation Internet service. The bandwidths of the backbone networks of APAN in 1998 were between $1 \mathrm{Mbps}$ and $35 \mathrm{Mbps}$. The US next generation Internet project "very high speed Backbone Network Service" (vBNS) already had $155 \mathrm{Mbps}$ bandwidth service in their backbone network. The operating policy of APAN was to provide high performance data transfer service, because, in 1998, it was still impossible to implement a huge bandwidth network in the Asia Pacific area. Now, the APAN network covers the Asia Pacific area, providing bandwidth between $45 \mathrm{Mbps}$ and 10 Gbps. In 2011, APAN became the none-profit organization and APAN strongly supports the research activities over the R\&E networks in Asia Pacific area.

\subsection{Trans-Eurasian Information Network 2 (TEIN2)}

TEIN or TEIN1 was an EU project that connected Europe and Korea. It started with a bandwidth of 10 Mbps. TEIN2 (Delivery of Advanced Network Technology to Europe [DANTE], n.d.) is a bit different from TEIN in that it has two goals. One is to provide an access network to Europe, and the other is to develop an interconnection network in the Southeast Asian area whose bandwidth is between $45 \mathrm{Mbps}$ and 1 Gbps. The main characteristic of the design topology is that it is not the star-shaped. That is, the TEIN2 network itself has its own backbone with connecting the four NOCs, and the number of routes for communicating between NOCs are more than two. It provides each Southeast Asian site with several routes to access the others. TEIN3 started in 2010 and TW, KH, LA, IN, LK, NP, PK, BD and BT (Tbl. 3) joined this activity.

\section{Background of R\&E network}

\subsection{R\&E network status in 2006}

In the old star-shaped R\&E network topology (Fig. 1) communication between the point sites had to go through the center of the star. Communication along these routes was frequently delayed due to the configuration. Now, however, the R\&E network community has grown, and the former point sites are now sometimes the center of a star. In some cases this growth generated several routes between two sites (Fig. 2). 


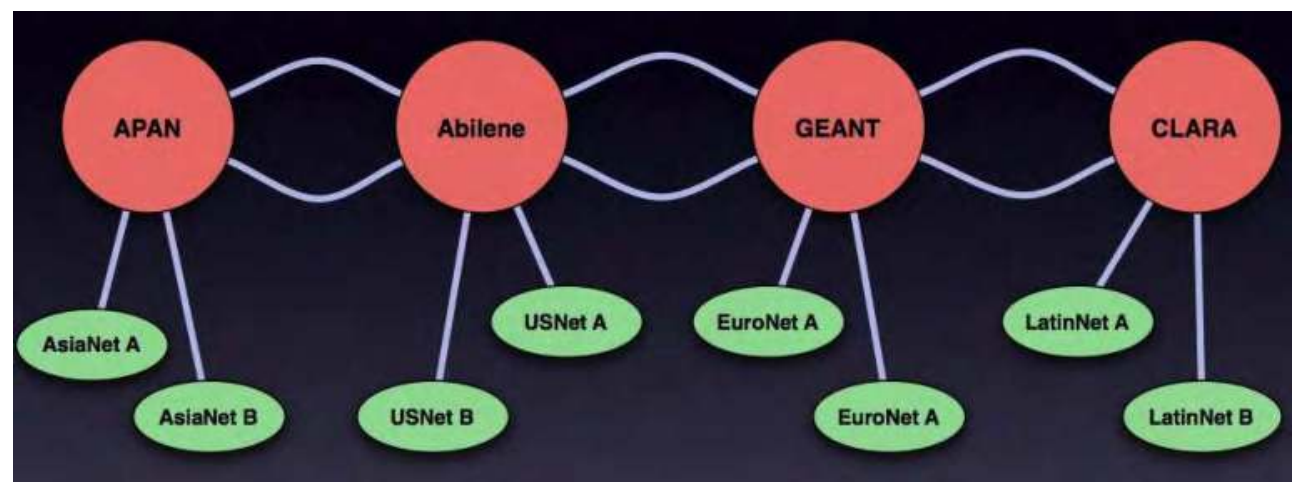

Fig. 1. Star shaped R\&E networks(Robb, 2006)

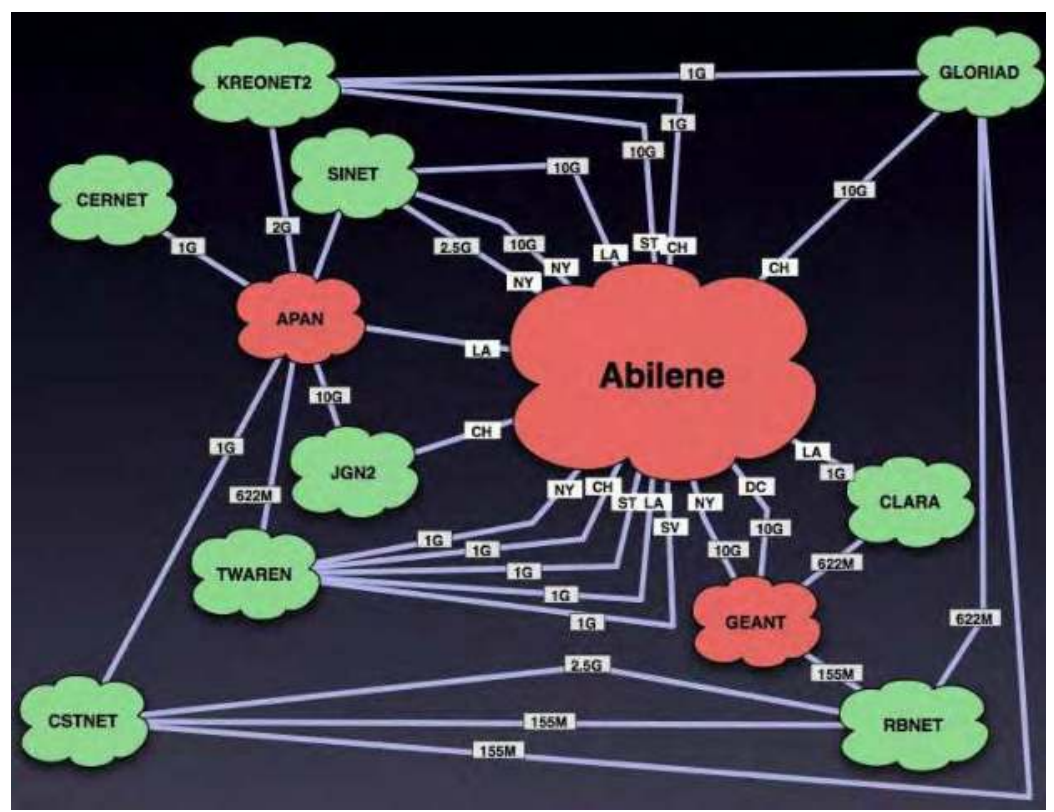

Fig. 2. Actual R\&E networks(Robb, 2006)

This complicated topology made the operation of the R\&E networks more difficult. To simplify operations, Research and Education Network Operators Group (RENOG), (Research and Education Network Operators Group [RENOG], n.d.) was developed and started reconfiguring the complicated and unstable routing. However, in the APAN area, the complicated routing unexpectedly worked and it was able to maintain high-speed communication with most of the network researchers, though with some delay. Network engineers like the word "redundancy" but most of the network engineers in the APAN area did not expect that the complicated topology and the complicated routing information would work so well in an emergency. 


\subsection{Before the earthquake}

The TEIN2 project started in 2006. Before that, the network topology style in Asia Pacific area was close to the star shape. Most of the networks in the APAN area started in Japan, and if a network did not start from Japan, it started from one of the point sites. That is why the star shape was kept. However, the TEIN2 topology does not look like a star. Even inside TEIN2, there are two or three routes to reach any other sites. An engineering meeting was held before TEIN2 started at which an agreement on routing policy seemed to have been reached. However, after TEIN2 started, there happened some of routing troubles in Asian Pacific area. A few troubles were from TEIN2 network directly but others were the routing advertise issues from the National Research and Education Networks (NRENs) of the TEIN2. To solve these problems, the operators held many meetings to develop tools. One tool summarized traffic conditions for each route (Asia-Pacific Advanced Network Japan [APAN-JP], n.d.). Another was a database to record preferable routes for each XP (Kurokawa, 2006). These were essentially monitoring and advising tools, not route configuration tools. Actual operations were done at the XPs based on the database, which was built by the database tool (Kurokawa, 2006). At the APAN Tokyo XP, the abstract of the routing policy was as follows.

- Communication with ID, SG, MY, TH, VN, CN, and AU (Tbl. 3) should be routed through TEIN2.

- Communication with PH should be direct.

- Communication with Micronesia should be routed through the Hawaii XP.

- Communication with TW should be direct.

- Generic communication with EU should be through US.

- Communication with US should be direct.

Figure 3 shows the one of the worst examples of communication between US university through KR. That is, if a university in US established communication with Merit or the University of Michigan, the packets were transferred through KR. In such a case, TCP-based applications often met with communication problems.

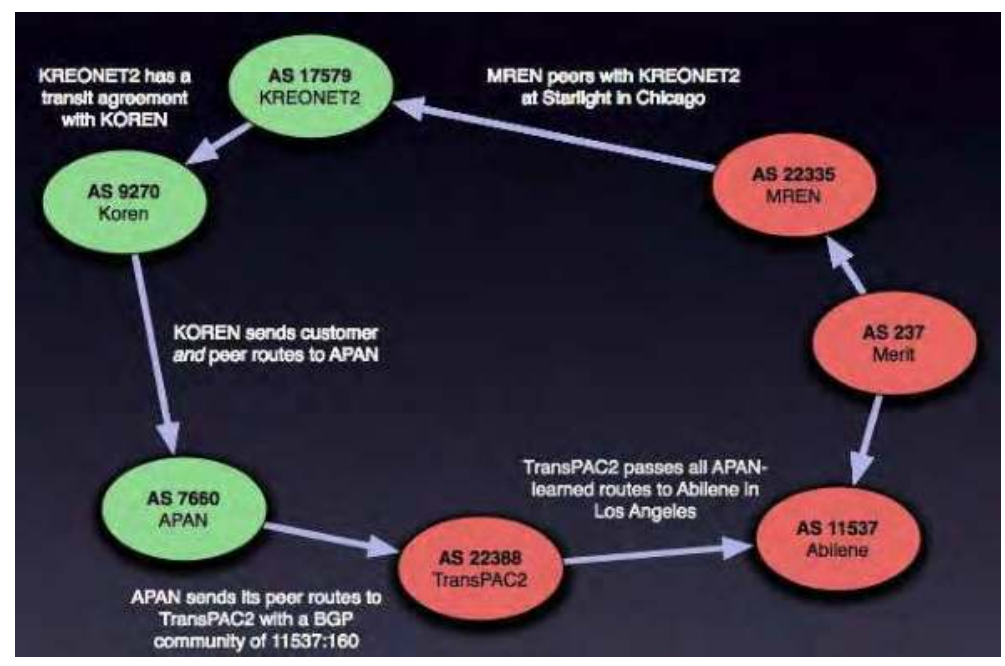

Fig. 3. Incorrect routing between universities in US through KR(Robb, 2006) 


\section{Network failures caused by Taiwan earthquake}

\subsection{Fiber breaks}

On December 26th, 2006 (UTC+9) there were two huge earthquakes near Taiwan. The first earthquake happened at 20:26 (UTC+8) (Academia Sinica, n.d.a), and the second one at 20:34 $($ UTC+8) (Academia Sinica, n.d.b). Fortunately, the earthquakes took place under the sea and the cities in TW were not heavily damaged, as happened in 1999. However, these two earthquakes did cause landslides over a wide area on the seabed near the Taiwan island. At 04:00 (UTC+9) on December 27th 2006, that is, after the second earthquake, the R\&E networks in the Asian area were shutdown. The cable companies investigated the reason for the lost connection and found that the earthquake had caused damage to the cable systems.

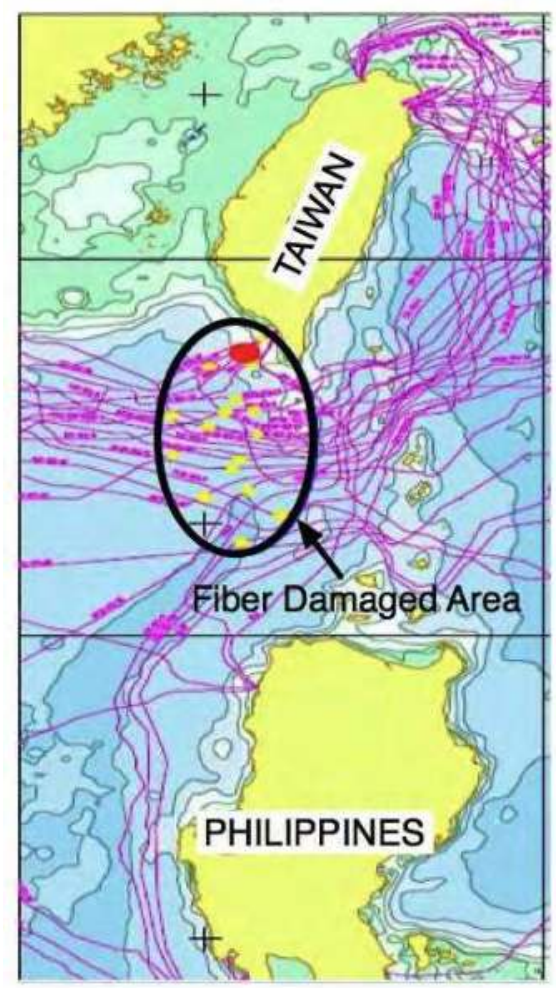

Fig. 4. Fiber cut area around Taiwan island (Konishi, 2007)

The circle in Fig. 4 shows the area where the cable systems were cut off. Most of the fiber cables in the eastern Asia area went through southwestern Taiwan. These cables were generally bought and shared by different telecom companies.

\subsection{Internet disconnections and lost BGP peerings}

After the earthquake, both commodity Internet traffic and R\&E traffic were cut off. For instance, the JP-PH, JP-CN, JP-SG, CN-US, HK-KR, TW-(HK+CN), and TW-SG connections were lost. That is, the R\&E network communities were divided into two groups. One was 
the group that consisted of JP, KR, TW, and US, and the other consisted of $\mathrm{CN}, \mathrm{HK}, \mathrm{VN}, \mathrm{MY}$, TH, SG, ID, and PH (Fig 5).

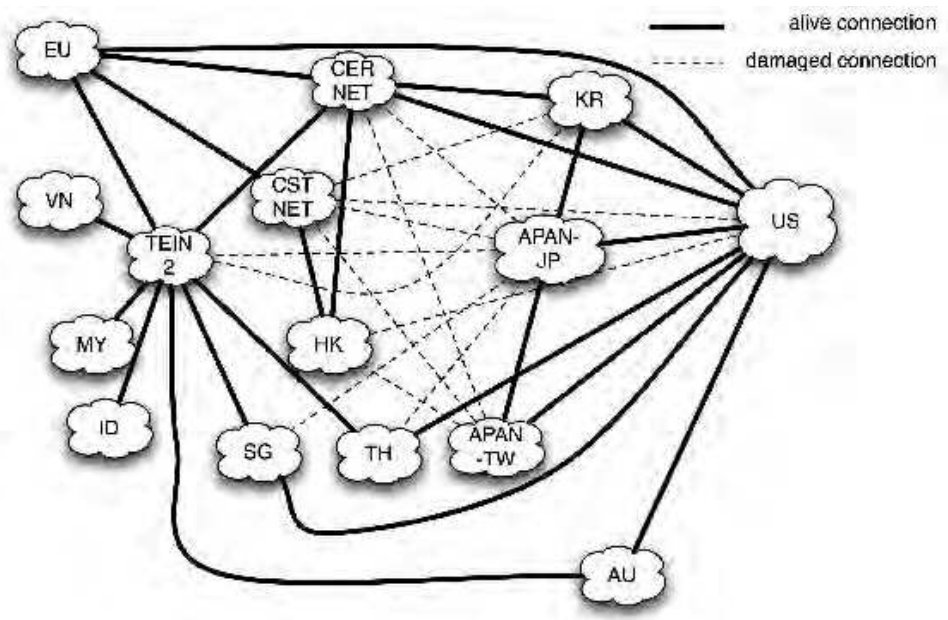

Fig. 5. Splitted R\&E networks in Asia Pacific area

The Internet disconnection occurred in the following order.

1. Link-layer disconnection because of the fiber cut

2. Lost primary BGP peerings

3. Automatic BGP re-routing along the alternative peer if any

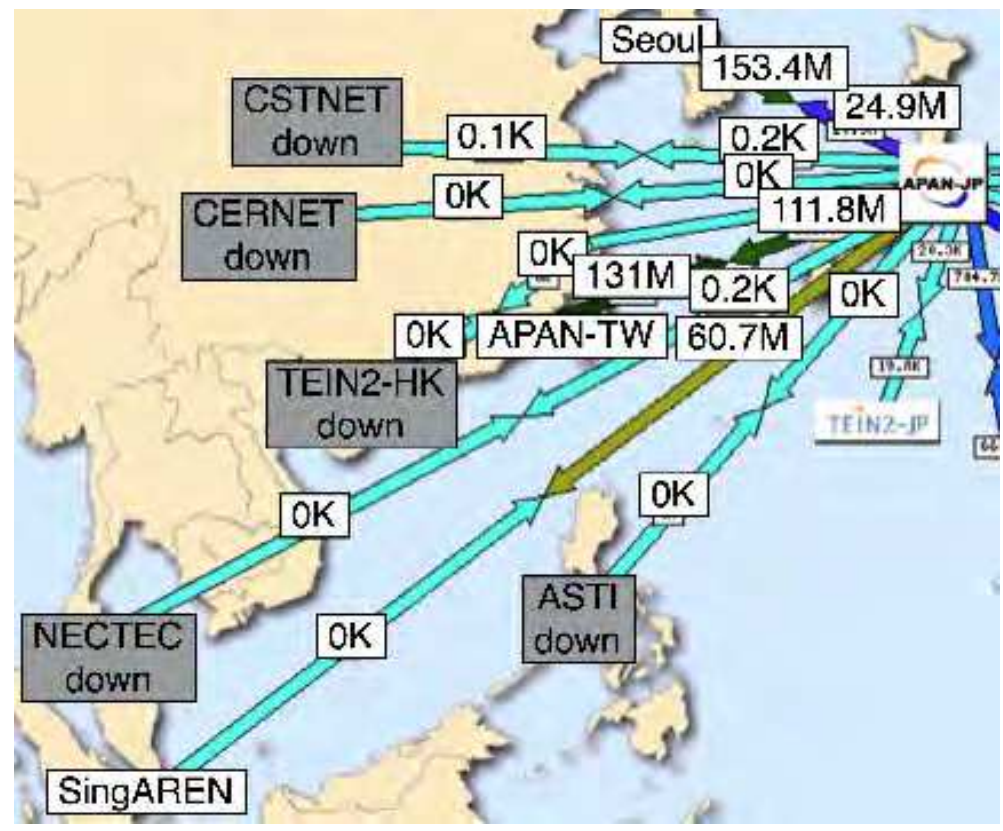

Fig. 6. Traffic weather map on Dec.27 2006 
Figure 6 shows the Internet traffic weather map at APAN Tokyo XP (APAN-JP, n.d.), which displays connectivity and link utilization in real time. In Fig. 6 it can be observed that the JPHK-CN, JP-TH, JP-SG, and JP-PH communications were lost and that there was $0 \%$ link utilization except a $100 \mathrm{Mbps}$ load between JP and KR.

The link-layer disconnection caused BGP sessions to expire. BGP peerings from JP to $\mathrm{HK}+\mathrm{CN}, \mathrm{TH}, \mathrm{SG}$, and $\mathrm{PH}$ were lost and automatically diverted to detour routes.

In general, when traffic is transferred to the detour AS routes, the traffic will flow along the longer AS path rather than the usual one, because the shortest AS path will be selected as the primary AS path according to BGP policy.

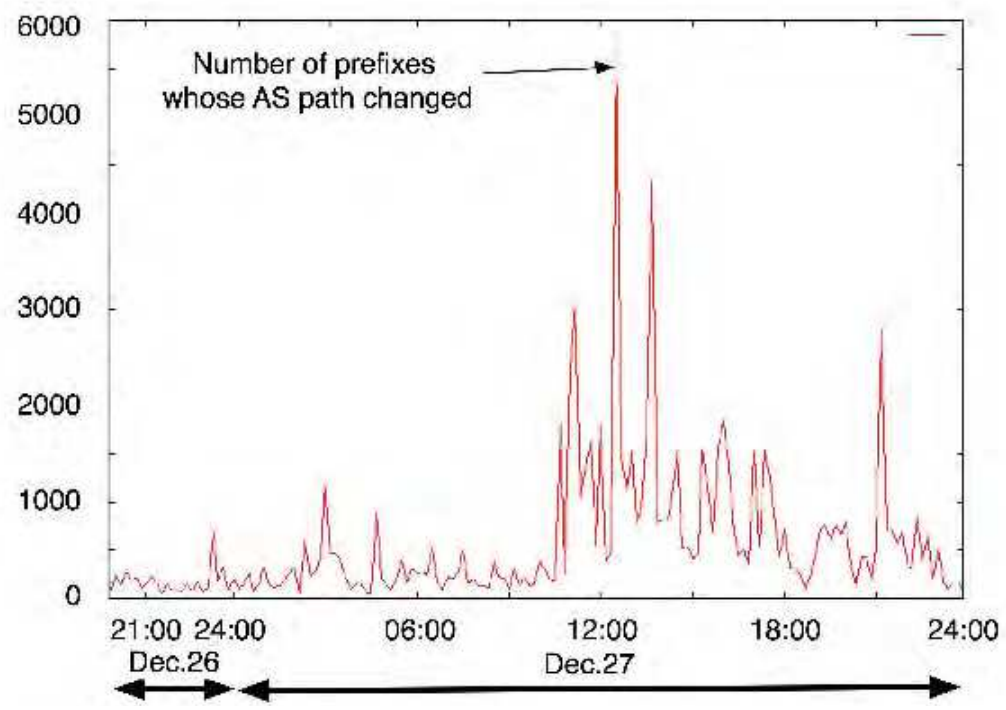

Fig. 7. Number of IP prefixes that experienced AS path changes (2006.12.26 - 2006.12.27 at QGPOP)

Figure 7 shows the AS path changes of each IP prefix observed just after earthquake from the QGPOP (Kyushu GigaPOP Project [QGPOP], n.d.) BGP router in JP. It can be seen in Fig. 7 that more than 1000 IP prefixes experienced AS path changes after the earthquake.

\subsection{Traffic load changes}

Fortunately, despite the earthquake, the CN-KR and KR-JP cables were unbroken. Therefore, we were able to observe the detour traffic along these links due to BGP rerouting.

Figure 8 shows the traffic between JP and KR on Dec. 27th, 2006. At about 04:30 (UTC+9) the inbound traffic pattern between JP and KR had changed dramatically. At the same time, as can be seen in Fig. 9, the traffic between JP and PH disappeared.

The reason for the traffic change could be inferred from the routing policy of APAN Tokyo XP. The route from CN to JP through KR was one of the lowest priority routes, but after the earthquake, it was chosen because there were no available BGP routes with high priorities. 


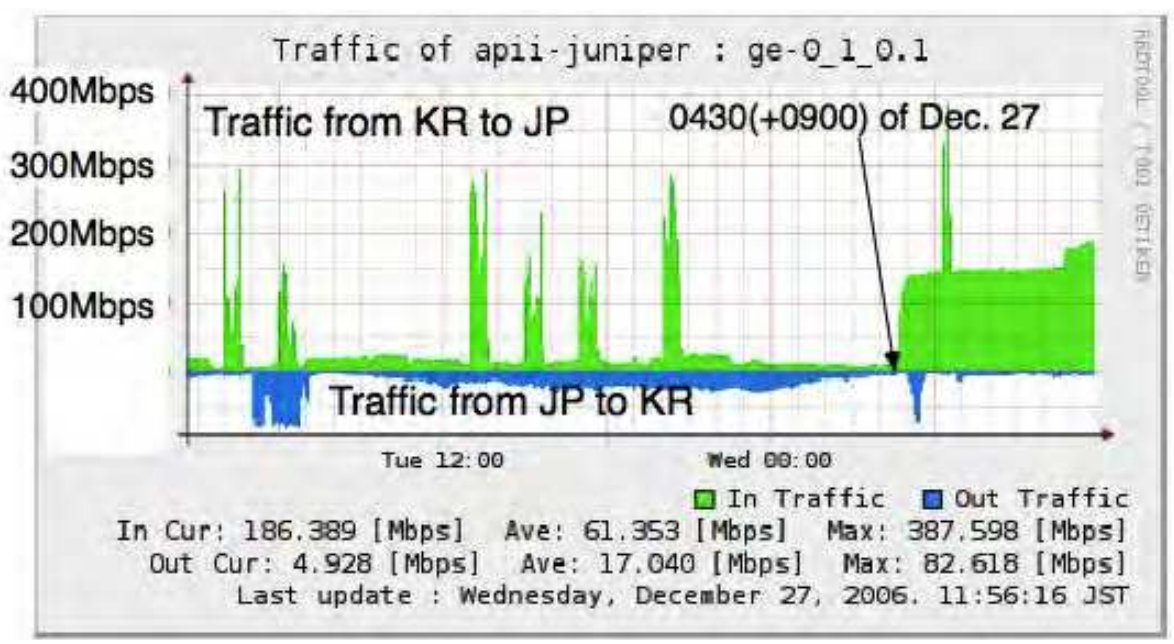

Fig. 8. Traffic between JP and KR on Dec.27 (UTC+9)\}

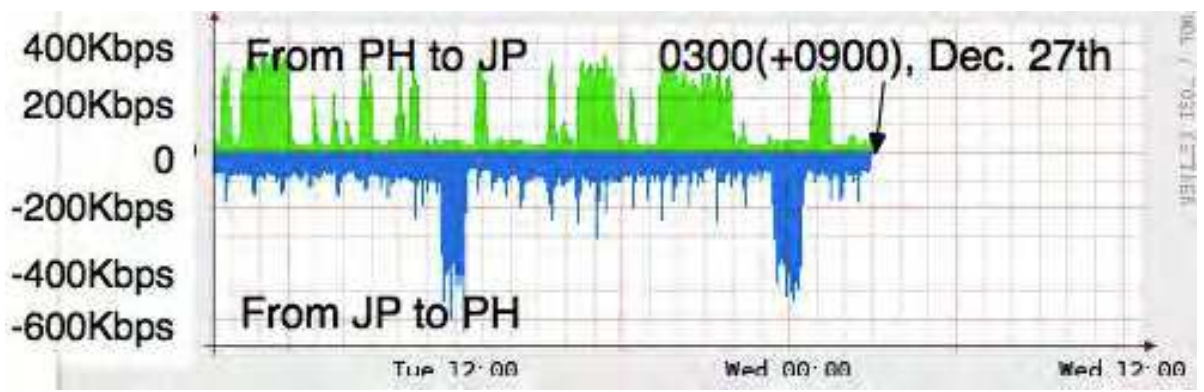

Fig. 9. Traffic between JP and PH on Dec.27 (UTC+9)

\subsection{Changes of BGP routing tables}

Table 1 shows the registered routing policy table of the Asia Pacific R\&E networks. It can be observed that ASTI (PH) lost connectivity to the R\&E networks, that CSTNET (CN) lost eastbound routes, and that APAN-JP (JP) lost connectivity to TEIN2.

CERNET (CN) had a direct connection to TW, but after the earthquake its connection was expected to be changed to the path through US, as shown in Fig. 10. But, the routing policy arrangement was different between JP and KR. The forward path between $\mathrm{CN}$ and TW chose the route through JP but the return path chose the route through US as shown in Fig. 11.

In addition, routing from JP to the "west" Asian networks (Fig. 5) was connected through US. The direct link between CSTNET $(\mathrm{CN})$ and US was also damaged.

Both CERNET and APAN Tokyo XP expect that the detour for JP-CN traffic should be through US, not through KR. Figure 10 shows the expected BGP route and the actual route between APAN-TW and CN. Since only APAN Tokyo XP implemented the strict routing policy, the $\mathrm{CN}$ traffic chose the shortest AS path. However, the traffic from JP to CN chose a routing policy that does not choose the route through $\mathrm{KR}$. 


\begin{tabular}{|c|c|c|c|c|c|c|c|c|c|c|c|c|}
\hline Src $\backslash$ Dst & APAN-I & CERNET & APAN- & KOREN & J ASTI & CSTNET & TEIN2 & SingAREN & j] UniNet & ThaiSARN & J|KREONET2 & AARNET \\
\hline & (JP) & $(\mathrm{CN})$ & \begin{tabular}{|l} 
TW \\
(TW)
\end{tabular} & (KR) & (PH) & $(\mathrm{CN})$ & & (SG) & $(\mathrm{TH})$ & (TH) & $(\mathrm{KR})$ & $(\mathrm{AU})$ \\
\hline $\begin{array}{l}\text { APAN- } \\
\text { JP }\end{array}$ & & D & direct & direct & $\mathrm{U}$ & C & $\mathrm{U}$ & direct & D & U & US & $\begin{array}{l}\text { US } \\
\text { Hawaii }\end{array}$ \\
\hline CERNET & $\mathrm{D}$ & & D & direct & $\mathrm{U}$ & N.A. & TEIN2 & TEIN2 & TEIN2 & TEIN2 & TEIN2 & TEIN2 \\
\hline \begin{tabular}{|l|} 
APAN- \\
TW \\
\end{tabular} & direct & D & & $\begin{array}{l}\text { APAN- } \\
\text { JP }\end{array}$ & U & U & N.A. & N.A. & N.A. & N.A. & APAN-JP & $\begin{array}{l}\text { APAN- } \\
\text { JP }\end{array}$ \\
\hline KOREN & direct & direct & $\begin{array}{l}\text { APAN- } \\
\text { JP }\end{array}$ & & U & $\mathrm{U}$ & TEIN2 & TEIN2 & TEIN2 & TEIN2 & direct & TEIN2 \\
\hline ASTI & $\mathrm{U}$ & $\mathrm{U}$ & $\mathrm{U}$ & $\mathrm{U}$ & & $\mathrm{U}$ & $\mathrm{U}$ & U & U & $\mathrm{U}$ & $\mathrm{U}$ & $\mathrm{U}$ \\
\hline CSTNET & C & N.A. & $\mathrm{U}$ & $\mathrm{U}$ & $\mathrm{U}$ & & N.A. & N.A. & N.A. & N.A. & $\mathrm{U}$ & N.A. \\
\hline TEIN2 & $\mathrm{U}$ & TEIN2 & N.A. & TEIN2 & $\mathrm{U}$ & N.A. & & TEIN2 & TEIN2 & TEIN2 & KOREN & TEIN2 \\
\hline & D: & & & th throp & $\mathrm{sht}$ & & & & & & achable & \\
\hline
\end{tabular}

Table 1. The monitored routing table for each R\&E network on Dec.27 2006 (Kurokawa, 2006)

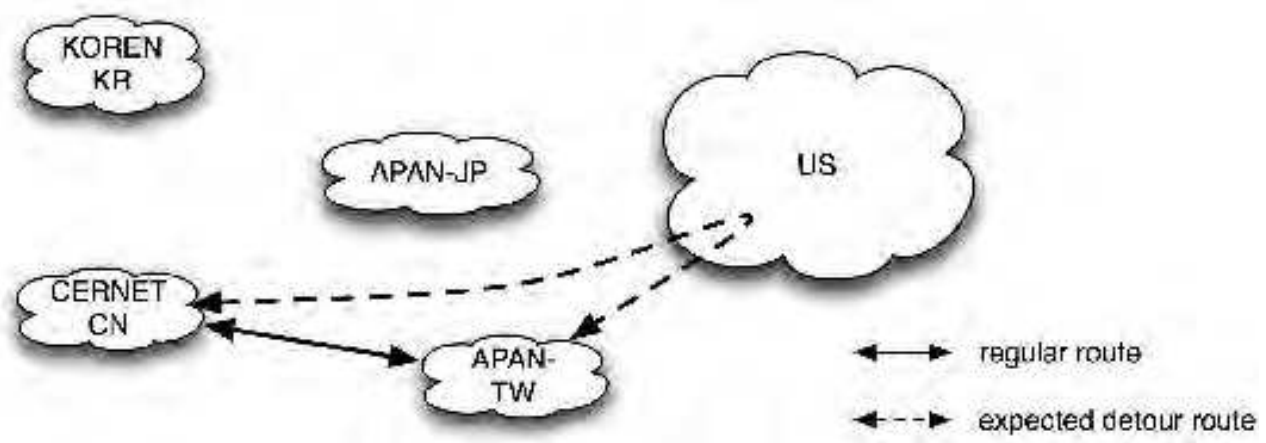

Fig. 10. BGP route changes of APAN before earthquake (Dec. 2006)

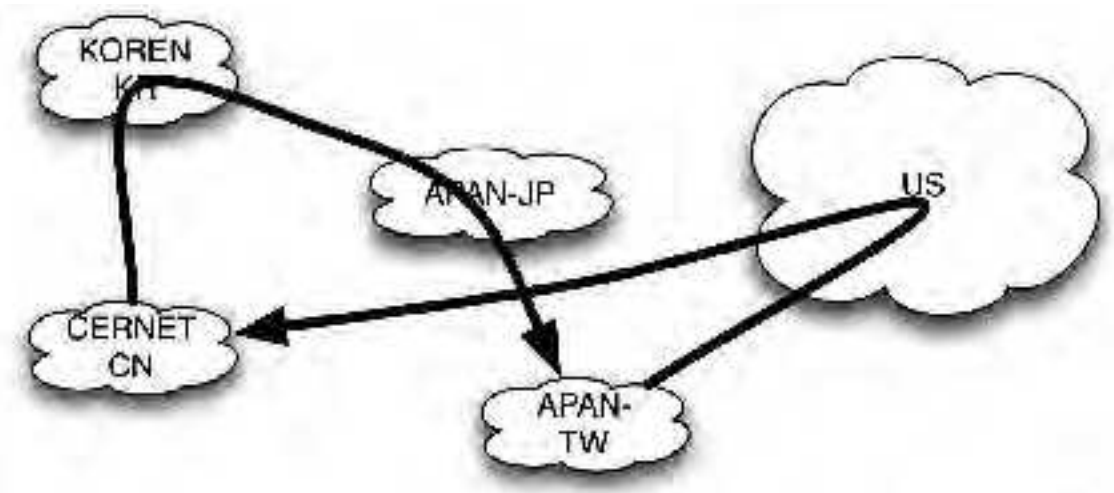

actual route

Fig. 11. BGP route changes of APAN after earthquake (Dec. 2006) 


\subsection{AS-level topology changes}

To investigate the BGP route changes in detail, we used an AS-topology visualization tool called "ABEL2" (Sakiyama et al., 2006) that utilizes BGP routing tables that are stored every 10 minutes.

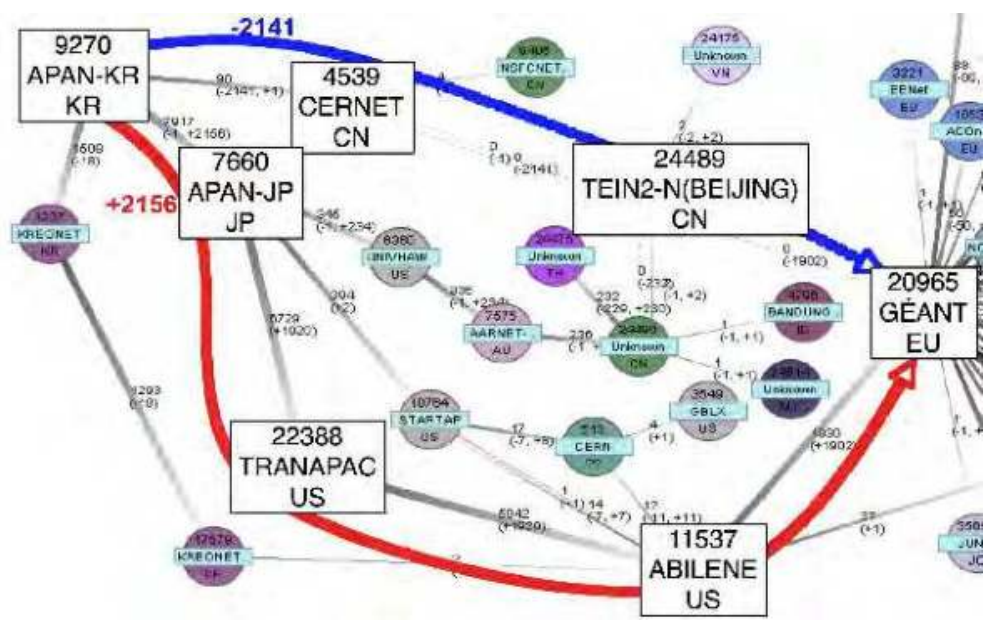

Fig. 12. After the earthquake at 19:30, Dec. $27(\mathrm{UTC}+9)$ 2006, the route from APAN-KR to GÉANT was diverted to a long AS path of (APAN-KR, APAN-JP, TransPAC, Abilene, GÉANT)

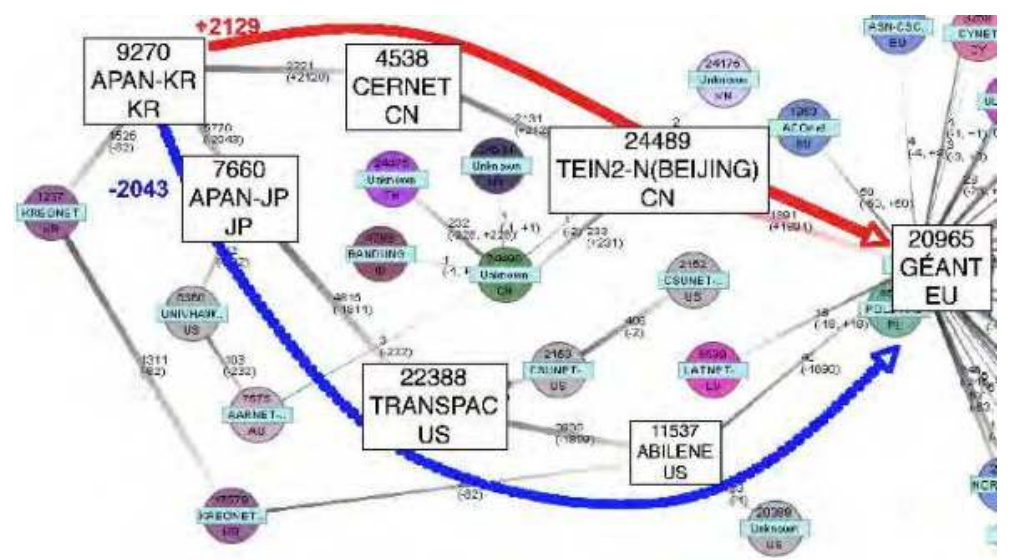

Fig. 13. Reconfigured AS path from APAN-KR to GÉANT (APAN-KR, CERNET, TEIN2-N, GÉANT) at 20:30, Dec. 27 (UTC+9) 2006

Figures 12, 13, and 14 show the changes between APAN-KR (AS9270) and GÉANT (AS20965) by the number of IP prefixes. It can be observed in Fig. 12, that just after the earthquake, at 19:30 on December 27th (UTC+9), 2006, the route from APAN-KR to GÉANT was diverted to the long AS path APAN-KR - APAN-JP - TransPAC (US) - 
Abilene (US) - GÉANT (Tbl. 4) because of the TEIN2 link outage. Therefore, to connect APAN-KR to GÉANT with a shorter AS path, the operator configured the BGP routing policy to make CERNET (CN) announce GÉANT prefixes. As shown in Fig. 13, the route from APAN-KR to GÉANT was switched through CERNET at 20:30 on Dec. 27th (UTC+9), 2006. At 20:50 on Dec. 17th, 2006 (UTC+9), the TEIN2-SG NOC announced the route to EU, too. Since the link bandwidth between SG and KR is larger than that between $\mathrm{CN}$ and $\mathrm{KR}$, the operator made a configuration for BGP routers to choose the AS path with the KR-SG link (Fig. 14).

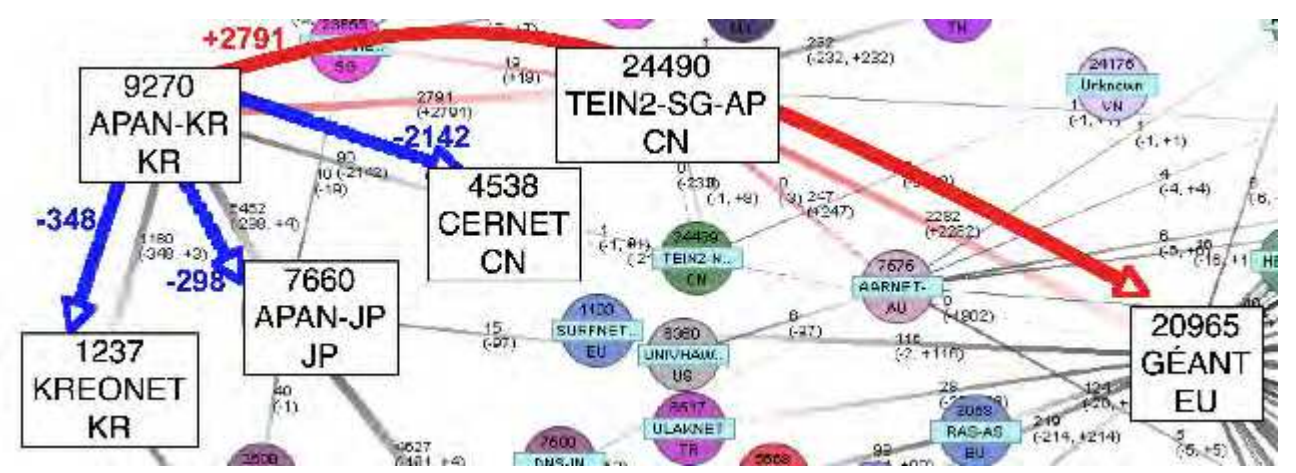

Fig. 14. Reconfigured AS path from APAN-KR to GÉANT through TEIN2-SG at 20:50, Dec. 27 (UTC+9) 2006

\subsection{Delay changes}

When Australia's Research and Education Network (AARNET) proposed that it would transit all the traffic of TEIN2 members (CN, SG, VN, MY, TH, ID), a flow data analysis was done and most of the traffic was found to be for Eastern Asia.

The APAN Tokyo XP and the TEIN2 NOC asked the AARNET NOC to route the TEIN2 traffic through Honolulu rather than through Seattle, because the traffic was between the Eastern Asia and TEIN2 members. The shorter Round Trip Time (RTT) worked better, especially with TCP-based applications (Tbl. 5). However, this operation occupied the link between Honolulu and Tokyo, because the bandwidth between Tokyo and Honolulu is 155 Mbps and the one between the Tokyo and TEIN2 NOCs is $622 \mathrm{Mbps}$ and the one between AARNET and TEIN2 is 1 Gbps (Fig. 15).

The link between KR and JP, whose bandwidth was $10 \mathrm{Gbps}$, was up. According to the analysis, the KR-JP link still had space to carry the traffic, and this route is much shorter than the route through Australia.

The TEIN2 and AARNET NOCs and the Tokyo XP stopped routing the TEIN2 traffic through Australia, and the KR transit policy was initiated. But the bandwidth between $\mathrm{CN}$ and KR was also $155 \mathrm{Mbps}$, too, so the link was occupied again (Fig. 16).

At that moment, the JGNII JP-SG link (Tbl. 4) was restored, but after precise check by the engineers, it looked as though the telecommunication company had switched the route and provided connectivity. TEIN2 NOC and the Tokyo XP decided to separate the traffic to Tokyo into two routes. That is, CN traffic was transferred through KR, and other TEIN2 traffic was transferred through SG (Fig. 17). 


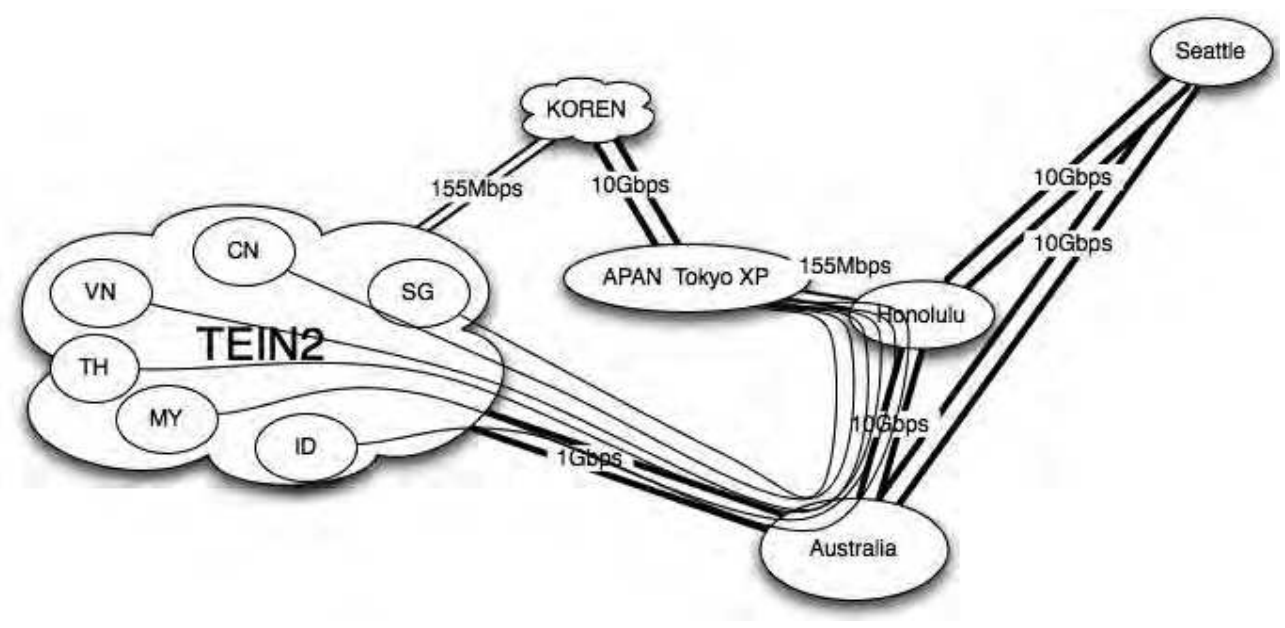

Fig. 15. TEIN2 traffic was carried through Hawaii.

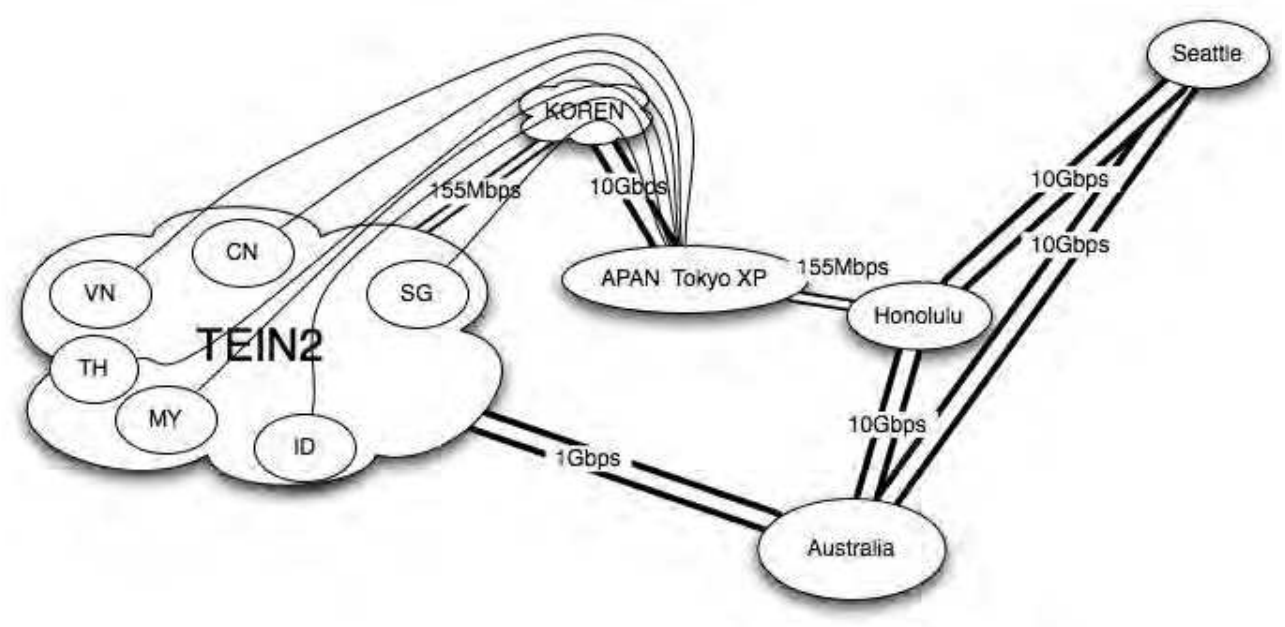

Fig. 16. TEIN2 traffic was routed through KR.

On December 27th after the fiber cut, automatic BGP re-routing has been carried out between SG and JP. The route from SG to JP became SG-AU-Hawaii-JP instead of the direct link and its RTT was increased to $426 \mathrm{~ms}$, while its normal RTT is around $88 \mathrm{~ms}$.

When the link between SG and KR was temporarily recovered with the backup fiber, the RTT values was $240 \mathrm{~ms}$ between SG and JP via KR on December 28th.

Finally, on January 12th when the link between JP and SG was recovered with the direct fiber, the RTT was reduced to $113 \mathrm{~ms}$, which is slightly increased than the usual case. 


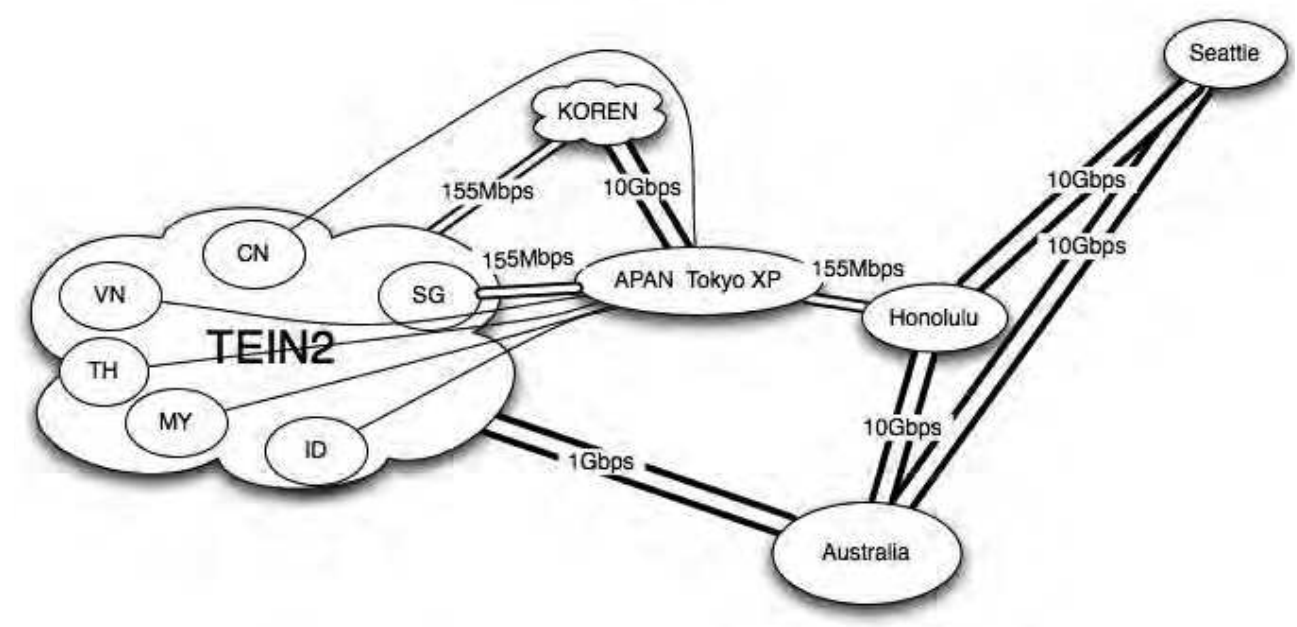

Fig. 17. TEIN2 traffic was routed through SG and KR.

Figure 18 shows the RTT between SG and JP just after the earthquake and the recovery process.

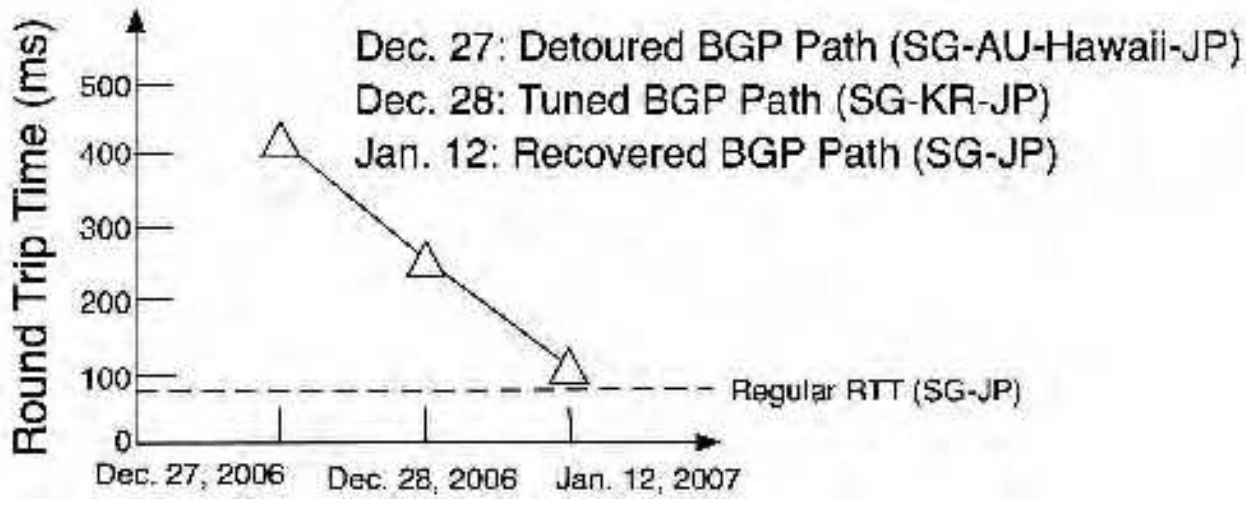

Fig. 18. RTT between SG and JP during the restoration process

\section{Network restoration methods}

After the network failures caused by the earthquake, several restoration steps were taken to restore communication. In this subsection, we discuss these steps.

\subsection{Automatic BGP re-routing}

Usually, the full BGP routing table includes a few "useless" routes (Tbl. 2). By "useless" we mean that the route itself provides only connectivity with the long RTT and insufficient bandwidth. Therefore, the network operators filter out such useless routes by setting the 
local preference to ignore them. However, after the earthquake, these useless BGP routes worked automatically as backup paths. In the Asia Pacific R\&E networks, the routes became very complicated after TEIN2 started because TEIN2 provided a few unexpected routes around the world. Because there were backup AS paths, automatic BGP re-routing could be used for first aid to provide the connectivity to the ASs that lost the primary paths. However, automatic BGP re-routing did not consider the traffic engineering parameters of the available bandwidth and the backup traffic load.

\begin{tabular}{|l|l|l|}
\hline (Src, Dst) & Usual Path & Useless Path \\
\hline (JP, TEIN2) & JP-TEIN2 & JP-Hawaii/Seattle-AU-TEIN2 \\
\hline (TEIN2, JP) & TEIN2-JP & TEIN2-GÉANT2-Abilene-JP \\
\hline (US, US) & Abilene & US-KREONET2-JP-TransPAC2-US \\
\hline (JP, KR) & JP-KR link & JP-TEIN2-JP- TEIN2-SG -AU-Seattle-KR \\
\hline
\end{tabular}

Table 2. Examples of the "useless routes"

\subsection{Traffic engineering with BGP policy change}

BGP by itself does not provide any information regarding link capacity or available bandwidth. Moreover, due to recent VLAN (Varadaraja, 1997) technology, the distance between two ASs has no relation to physical distance. Thus, QoS information of the detour routes must be examined by the operators. This makes systems reliant on human knowledge of traffic engineering. To remove the congestion due to the long detour AS path, we changed the BGP routing policy as shown in Fig. 13 and Fig. 14.

The members of TEIN2 (VN, MY, SG, ID, PH) lost their connections to APAN Tokyo XP because the fiber broke. AARNET NOC proposed backup routes for accessing APAN Tokyo $\mathrm{XP}$ through AU and Hawaii. However, this solution caused congestion on both the CN-KR and Hawaii-JP links. Besides, $\mathrm{CN}$ traffic took an asymmetrical path.

Therefore, to solve the traffic engineering issue, Tokyo XP made a decision to divide $\mathrm{CN}$ traffic by announcing CN IP prefixes through KR NOC and grouping CN prefixes at Tokyo $\mathrm{XP}$. The results were monitored by Cisco NetFlow (CISCO, n.d.). The operators found out that half of the KR traffic was from $\mathrm{CN}$. After a careful examination, it was discovered that a part of the CN traffic was from CERNET but the other part was from TEIN2.

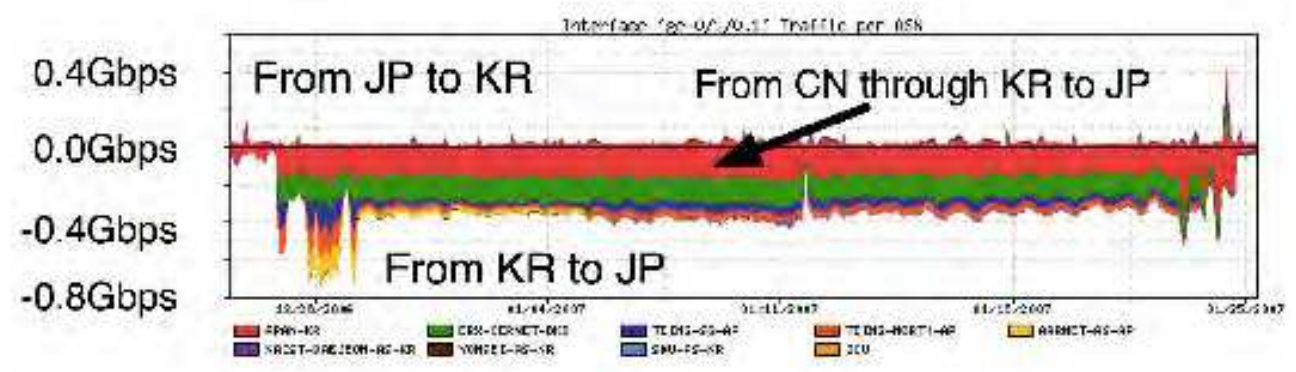

Fig. 19. Traffic from KR monitored at APAN JP (Dec. 262006 - Jan. 26 2007)\} 
Figure 19 shows the traffic load for each source AS. Although the total traffic is about 0.4 Gbps, the real KR traffic was about $0.2 \mathrm{Gbps}$. $0.1 \mathrm{Gbps}$ is occupied by CERNET traffic and the rest by TEIN2.

\subsection{Port reconfiguration}

In spite of the recovery steps, a few sites that had only single-link connections to the Internet could not directly reach the R\&E networks. Therefore, after the fiber was fixed, a few singlelink sites had the connections via Internet commodity service. To fix this problem, the operators had to change the port configuration at switches that were able to provide high performance connections.

Two days after the earthquake, the link between KR and SG was restored and SG started making routing announcements to TEIN2 members (ID, SG, TH, MY). However, the link between SG and KR was not the original fiber, and its RTT increased greatly because a detour route was assigned. Similarly, the direct link between $\mathrm{PH}$ and JP was replaced with a detour route with a long RTT. When an online demonstration was being prepared for the 2007 APAN meeting held in PH between January 22nd and 26th 2007, the restored JP-PH link had a very long RTT because it went through mainland China, as shown in Fig. 20.

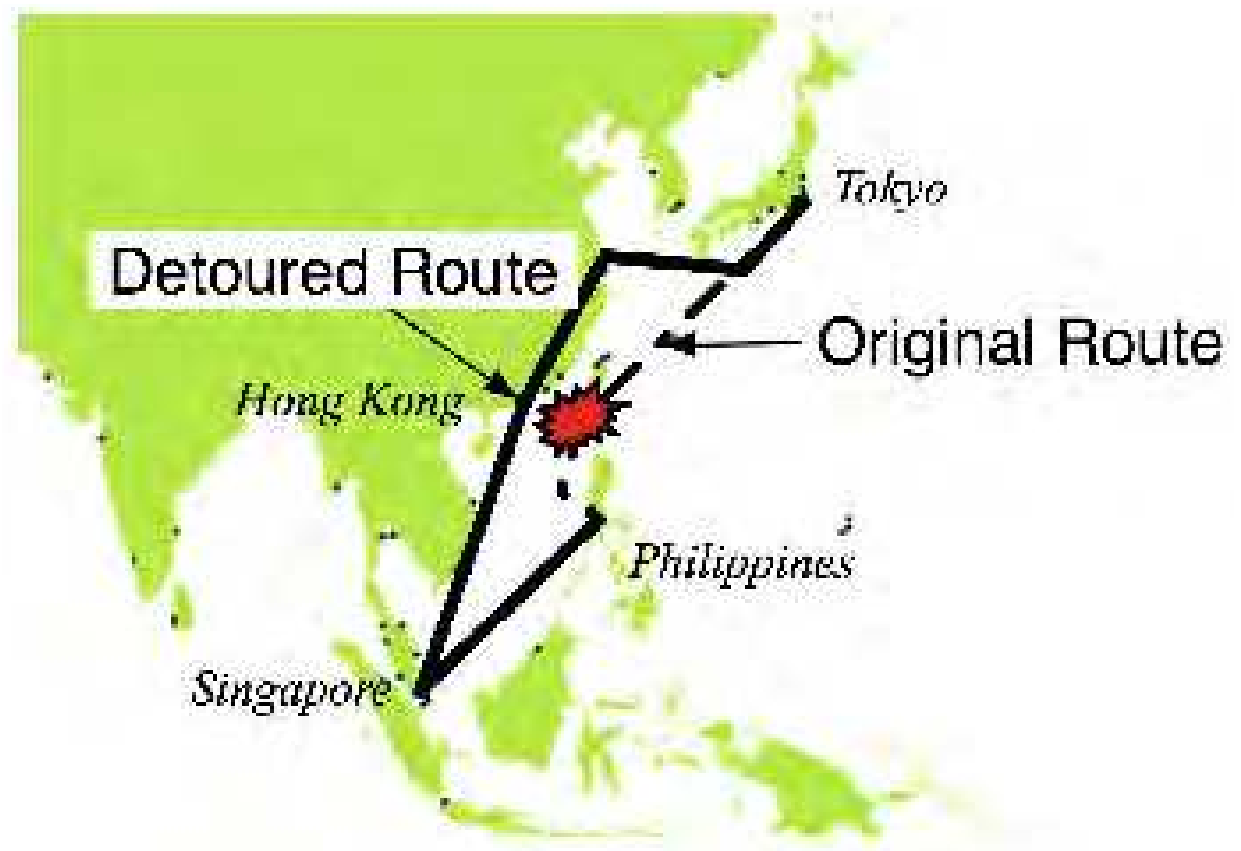

Fig. 20. JP-PH detour route circuit

During the 23rd APAN meeting demonstration, the Prince of Wales Hospital at the Chinese University of Hong Kong (CUHK) was expected to join. However, its IP prefix was announced only by CSTNET. Thus, it was only reachable over a commodity Internet link with a small bandwidth. To solve this problem, APAN Tokyo XP changed the port configuration of fibers. That is, the CSTNET fiber for accessing APAN Tokyo XP was 
plugged to the TEIN2-HK router. Finally, CUHK was directly connected to TEIN2. Finally, with this solution, CUHK and CSTNET were supplied with huge-bandwidth and short-RTT connections to the R\&E network.

\section{Lessons}

From the process of recovering from network failures across several ISP's in Asia Pacific R\&E networks, we encountered several network management challenges especially regarding fault management. In this subsection, we describe the lessons learned during the recovery operations.

\subsection{Fault-tolerant fiber topology design}

BGP routing policies are usually made to avoid asymmetric or useless AS paths by setting the appropriate local preference values. However, these alternative AS paths worked as backup paths. Before the network failures from earthquake, Asia Pacific R\&E network operators thought that removing the useless routes was urgent, because routing became too complicated after TEIN2 started. However, this complicated routing was able to provide valuable connections during network failures. This shows that maintaining full-mesh style routing information is very important for fault-tolerant routing.

Though BGP re-routing over the redundant AS paths was successful for the first step in restoration, it was not sufficient to provide full backup service without congestion by considering the traffic load. Since BGP routing does not carry QoS information, such as link capacity, link utilization, or available bandwidth, traffic re-routed to the backup AS path had experienced poor QoS, such as long delays. Therefore, QoS-aware BGP routing or traffic engineering-aware BGP routing is necessary.

\subsection{Integrated network management}

During the restoration process, we used various network monitoring tools such as an MRTG (Oetiker, n.d.a), a network weather map, a BGP routing table visualizer, and a flow monitor. At first, the link outage was noticed on the network weather map, and the abrupt change of traffic load was noticed on the MRTG. However, the fast fault detection method that encompasses physical, link, routing, and application layers is necessary because it was able to identify the exact failure points and visualize their impacts on the network. In addition, a simulator or emulator that could show the results with the network topology and the traffic load before and after failures would be very useful in predicting the effects of faultmanagement decisions. While we took various restoration steps, we had to process the information collected by each different network-monitoring tool. Finally, the operators interpreted the situation and implemented recovery decisions manually. If the iperf (GOOGLE, n.d) or bwctl (INTERNET2, n.d.) is available throughout the network, the endto-end available bandwidth between ASs can be easily estimated. For example, to access Sydney from Tokyo, there are two possible routes. One is Tokyo - Seattle - Sydney, and the other is Tokyo - Honolulu - Sydney. The former provides $10 \mathrm{Gbps}$ but has a long RTT. The latter route includes a bottleneck along the $155 \mathrm{Mbps}$ path but has a short RTT. In addition, to make the final decision, we had to check the flow data, because MRTG or RRDTool (Oetiker, n.d.b) do not classify traffic breakdowns by their source/destination ASs. When the traffic from KR increased suddenly, the operators could not understand the reasons. This shows that integrated network monitoring or management systems would be very 
useful for collecting information from several independent monitoring systems and for providing the correct information in an integrated wide view in case of significant network failures.

\subsection{Emergency communication between operators}

After the earthquake, communication among NOCs was difficult because the fiber break disrupted VoIP and legacy telephone service. Moreover, the earthquake happened on December 26th 2006, overlapping with the Christmas holiday. Thus, all the communication was routed over the instant messaging system and e-mails were routed over the detoured network even though it provided poor quality service. It became obvious that the emergency communication should be guaranteed in case of failures so that the recovery process can be started quickly

\section{Summary}

Since the Internet continues to grow globally and becomes ever more important in daily life, business, and research, the need for fault-tolerant service in network management becomes more urgent. However, during the network failures caused by the 2006 earthquake, it was shown that there are still many challenges in fault-tolerant network management research. Even though multiple fiber cores are installed together to provide backup service, they may be useless during severe natural disasters. Therefore, full-mesh or fiber-disjoint physical network topology should be designed for use during failures. On the available topology, it was seen that BGP routing provided backup AS paths, which was useful for the first step in restoration. However, the traffic engineering issues during restoration were difficult to solve because all the information, such as link capacity, available bandwidth, link delay, traffic load, and routing policy, had to be collected, interpreted, and acted on by human operators. In spite of BGP re-routing, we had to deal with a few single-link ASs to establish direct connections to the R\&E networks. From this experience of network recovery during a significant natural disaster affecting several different countries and ISP's, we were able to gather valuable information on network management during emergencies. Therefore, in the Internet of the future, designers should focus on fault-tolerant network management study including robust physical topology, cross-layer restoration, traffic engineering combined with BGP routing, and simulation of failures in the network.

We would like to show the one example from this lesson. In August 2009, the earthquake happened again near Taiwan island and the fiber cut happened again. Table 1 tool was improved as "Compath" already and the table was constructed again for this disaster (Kurokawa, 2010). At that time, the medical demonstration was planned in Asian area and the connectivity of the Thailand was required. The compath table recommended that the TW should have become the hub for the southeast Asian area. This was against the policy of TEIN2, but the R\&E networks object is to support the researchers and such the flexibility was approved and the tool from the lesson of the 2006 earthquake improved the network operations.

\section{Acknowledgment}

After the earthquake, KDDI OTC (Otemachi Technical Center) worked very hard to fix the routing problems of APAN Tokyo XP both in their capacity as paid staff and as volunteers. 
Yutaka Watanabe, OTC's director, took the MRTG snapshot of Figs. 8 and 9. AARNET NOC offered us the backup routes to access the TEIN2. KOREN NOC worked very hard to keep communication open between CERNET and ASNET even during the holiday. Hawaii University NOC worked very hard to keep control of this complicated routing. KDDI investigated the reason for the communication failures in the APAN area and gave useful advice to APAN Tokyo XP. The staff of Genkai XP NOC accepted CERNET's traffic from KOREN by upgrading the JP-KR link bandwidth. Due to these great efforts and the collaboration among the network engineers, we were able to quickly restore the Asia Pacific R\&E networks.

This research was supported by the MIC (Ministry of Information and Communication), Korea, under the ITRC (Information Technology Research Center) support program supervised by the IITA (Institute of Information Technology Assessment) (NIPA-2011C1090-1131-0005).

\section{Appendix}

\begin{tabular}{|l|l|}
\hline Country code & Name \\
\hline JP & Japan \\
\hline TH & Thailand \\
\hline MY & Malaysia \\
\hline HK & Hong Kong \\
\hline ID & Indonesia \\
\hline SG & Singapore \\
\hline TW & Taiwan \\
\hline CN & China \\
\hline US & United States of America \\
\hline KR & Korea \\
\hline VN & Vietnam \\
\hline PH & The Philipppines \\
\hline NP & Nepal \\
\hline AU & Australia \\
\hline BD & Bangladesh \\
\hline IN & India \\
\hline LK & Sri Lanka \\
\hline BT & Bhutan \\
\hline KH & Cambodia \\
\hline LA & Laos \\
\hline PK & Pakistan \\
\hline
\end{tabular}

Table 3. ISO 3166 Country Code (International Organization for Standardization [ISO], n.d.) in this chapter 


\begin{tabular}{|c|c|c|}
\hline Network Name & Description & Area \\
\hline AIII & $\begin{array}{l}\text { Asian Internet } \\
\text { Interconnection Initiatives }\end{array}$ & $\begin{array}{l}\text { JP, VN, PH, TH, MY, SG, } \\
\text { ID, NP }\end{array}$ \\
\hline APAN & $\begin{array}{l}\text { Asia-Pacific Advanced } \\
\text { Network }\end{array}$ & $\begin{array}{l}\text { JP, KR, CN, TW, HK, VN, } \\
\text { TH, MY, SG, AU, NZ, PK, } \\
\text { IN, KL, BD }\end{array}$ \\
\hline TEIN2 & $\begin{array}{l}\text { Trans-Eurasia Information } \\
\text { Network } 2\end{array}$ & $\begin{array}{l}\text { JP, CN, VN, PH, TH, MY, } \\
\text { SG, ID, AU, EU }\end{array}$ \\
\hline TEIN3 & $\begin{array}{l}\text { Trans-Eurasia Information } \\
\text { Network } 3\end{array}$ & $\begin{array}{l}\text { AU, BD, BT, KH, CN, IN, } \\
\text { ID, JP, KR, LA, MY, NP, } \\
\text { PK, PH, SG, LK, TW, TH, } \\
\text { VN }\end{array}$ \\
\hline ASTI & $\begin{array}{l}\text { Advanced Science and } \\
\text { Technology Institute, the } \\
\text { link owner of PREGINET }\end{array}$ & $\mathrm{PH}$ \\
\hline PREGINET & $\begin{array}{l}\text { Philippine Research } \\
\text { Education and Government } \\
\text { Information Network }\end{array}$ & $\mathrm{PH}$ \\
\hline CSTNET & $\begin{array}{l}\text { China Science \& Tecnology } \\
\text { Network }\end{array}$ & $\mathrm{CN}$ \\
\hline CERNET & $\begin{array}{l}\text { China Education and } \\
\text { Research Network }\end{array}$ & $\mathrm{CN}$ \\
\hline GÉANT & $\begin{array}{l}\text { R\&E Network, the AS } \\
\text { registration name }\end{array}$ & Europe \\
\hline GÉANT2 & R\&E Network & Europe \\
\hline KOREN & $\begin{array}{l}\text { Korea Advanced Research } \\
\text { Network }\end{array}$ & KR \\
\hline SingAREN & $\begin{array}{l}\text { Singapore Advanced } \\
\text { Research and Education } \\
\text { Network }\end{array}$ & SG \\
\hline UniNet & inter University Network & $\mathrm{TH}$ \\
\hline ThaiSARN & $\begin{array}{l}\text { Thai Social/Scientific } \\
\text { Academic and Research } \\
\text { Network }\end{array}$ & $\mathrm{TH}$ \\
\hline KREONET2 & $\begin{array}{l}\text { Korea Research } \\
\text { Environment Open Network } \\
2 \text {, the domestic name is } \\
\text { KREONET }\end{array}$ & $\mathrm{KR}$ \\
\hline AARNET & $\begin{array}{l}\text { Australia's Research and } \\
\text { Education Network }\end{array}$ & $\mathrm{AU}$ \\
\hline QGPOP & Kyushu Gigapop & $\mathrm{JP}$ \\
\hline JGNII & Japanese R\&E Network & JP, US, TH, SG \\
\hline
\end{tabular}

Table 4. Network Name 


\begin{tabular}{|c|c|c|c|}
\hline \multicolumn{4}{|c|}{ OSI Model } \\
\hline & Data unit & Layer & Function \\
\hline \multirow{4}{*}{$\begin{array}{l}\text { Host } \\
\text { layers }\end{array}$} & \multirow[t]{3}{*}{ Data } & 7. Application & Network Process to application \\
\hline & & 6. Presentation & $\begin{array}{l}\text { Data representation and } \\
\text { encryption }\end{array}$ \\
\hline & & 5. Session & Interhost communication \\
\hline & Segment/Datagram & 4. Transport & $\begin{array}{l}\text { End-toend connections and } \\
\text { reliability }\end{array}$ \\
\hline \multirow[t]{3}{*}{$\begin{array}{l}\text { Media } \\
\text { layers }\end{array}$} & Packet & 3. Network & $\begin{array}{l}\text { Path determination and logical } \\
\text { addressing }\end{array}$ \\
\hline & Frame & 2. Data Link & $\begin{array}{l}\text { Physical addressing (MAC \& } \\
\text { LLC) }\end{array}$ \\
\hline & Bit & 1. Physical & $\begin{array}{l}\text { Media signal and binary } \\
\text { transmission }\end{array}$ \\
\hline
\end{tabular}

Table 5. Open Systems Interconnection (OSI) Basic Reference Model

\section{References}

Academia Sinica, (n.d.). The December 26, 2006, Pingtung, Taiwan earthquake, In : Academia Sinica, 26.07.2011, Available from :

http:/ /www.earth.sinica.edu.tw/ smdmc/recent/2006/200612261226.htm

Academia Sinica, (n.d.). The December 26, 2006, Pingtung, Taiwan earthquake, In : Academia Sinica, 26.07.2011, Available from :

http://www.earth.sinica.edu.tw/ smdmc/recent/2006/200612261234.htm

Asian Internet Interconnection Initiative Project, (n.d.). Asian Internet Interconnection Initiative Project, In: Asian Internet Interconnection Initiative Project, 26.07.2011, Available from : http://www.ai3.net/

Asia-Pacific Advanced Network, (n.d.). Welcome to Asia-Pacific Advanced Network, In: Asia-Pacific Advanced Network, 26.07.2011, Available from: http:/ /www.apan.net/

Asia-Pacific Advanced Network Japan, (n.d.). APAN-JP Network Traffic Weathermap, In : APAN Japan Consortium, 26.07.2011, Available from : http://monitor.jp.apan.net/weathermap/

CISCO, (n.d.). Cisco IOS NetFlow In : Cisco, 26.07.2011, Available from : http://www.cisco.com/en/US/products/ps6601/products_ios_protocol_group_h ome.html

Delivery of Advanced Network Technology to Europe, (n.d.). TEIN2, In: DANTE, 26.07.2011, Available from: http:/ / www.tein2.net/

Fumagalli, A. \& Valcarenghi, L. (2000). IP Restoration vs. WDM Protection: Is There an Optimal Choice?, IEEE Network Magazine, Vol. 14, No. 6, (December 2000), pp. 3441, ISSN 08908044

Gerstel, O. \& Ramaswami, R. (2000), Optical Layer Survivability: a Services Perspective, IEEE Communications Magazine, Vol. 38. No. 3, (March 2000), pp. 104-113, ISSN 01636804

GOOGLE, (n.d.). iperf - TCP and UDP bandwidth performance measurement tool, In: Google Project Hosting, 26.07.2011., Available from :

http://code.google.com/p/iperf/ 
Hanks, T. \& Kanamori, H. (1979). A Moment Magnitude Scale, Journal of Geogphysical Research, Vo. 84, No. B5, (May 1979), pp. 2348-2350, ISSN 0148-0227

Iannaccone, G. ; Chuah, C. ; Mortier, R. ; Bhattacharyya, S. \& Diot, C. (2002). Analysis of Link Failures in an Backbone, ACM Internet Measurement Conference, Marseille, 2002

International Organization for Standardization, (n.d.). ISO - Maintenance Agency for ISO 3166 country codes - English country names and code elements In : International Organization for Standardizaation, 26.07.2011, Available from :

http://www.iso.org/iso/en/prods-services/iso3166ma/02iso-3166-code-lists/listen1.html

INTERNET2, (n.d.). Bandwidth Test Controller (BWCTL), In: Internet2, 26.07.2011, Available from : http:/ / e2epi.internet2.edu/bwctl

Konishi, K. (2007). Cables restoration, 23rd APAN Manila Meeting, Manila, Feburary 2007

Kurokawa, Y. (2006). Registration of Policy Routing Choices, 22nd APAN Singapore Meeting , Singapore, June 2006

Kurokawa, Y. (2010). Utilization of Compath Tool in Multiple Submarine Cables were cut off near Taiwan on August, 2009, 29th APAN Sydney Meeting, Australia, February 2010

Kyushu GigaPOP Project, (n.d.). QGPOP (Kyushu GigaPOP Project), In: QGPOP (Kyushu GigaPOP Project), 26.07.2011, Available from: http://www.qgpop.net

Lougheed, K. \& Rekhter, Y. (1989), Border Gateway Protocol (BGP), In: RFC 1105, 26.07.2011, Available from : http:// datatracker.ietf.org/doc/rfc1105/

Markopoulou, A. ; Iannacccone, G. ; Chuah, C. ; Mortier, R. ; Bhattacharyya, S. \& and Diot, C. (2004). Characterization of Failures in an IP Backbone, IEEE Infocom, Hong Kong, 2004

Oetiker, T. (n.d.). MRTG - Tobi Oetiker's MRTG - The Multi Router Traffic Grapher, In : MRTG, 26.07.2011. Available from : http://oss.oetiker.ch/mrtg/

Oetiker, T. (n.d.), RRDtool - About RRDtool In : RRDtool - About RRDtool, 26.07.2011, Available from : http://oss.oetiker.ch/rrdtool

Ramamurthy, S. ; Sahasrabuddhe, L. \& Mukherjee, B. (2003). Survival WDM Mesh Networks, IEEE/OSA Journal of Lightwave Technology , Vol. 21, No. 4, (April 2003), pp. 870-883, ISSN 0733-8724

Research and Education Network Operators Group, (n.d.) In : RENOG, 26.07.2011, Available from : http://www.renog.org

Robb, C. (2006). Global Research and Education Network Routing Issues, 2006 Spring Internet2 Member Meeting, Arlington VA, April 2006

Saharabuddhe, L. ; Ramanurthy, S. \& Mukherjee, B. (2002). Fault Management in IP-OverWDM Networks: WDM Protection vs. IP Restoration, IEEE Journal on Selected Areas in Communications, Vol. 20, No. 1, (January 2002), pp. 21-33, ISSN 0733-8716

Sakiyama, R. ; Okamura, K. \& Lee, Y. (2006). Visualization of Temporal Difference of BGP Routing Information, APAN Network Research Workshop, Singapore, Singapore, June 2006

Sharma, V. \& Hellstrand, F. (month 2003). Framework for Multi Protocol Label Switching (MPLS)-based Recovery, In : RFC 3469, 26.07.2011, Available from: http://datatracker.ietf.org/doc/rfc3469/

Varadarajan, S. (1997). Virtual Local Area Networks , In: Virtual Local Area Networks, 26.07.2011., Available from :

http://www.cs.wustl.edu/ jain/cis788-97/ftp/virtual_lans/index.htm 


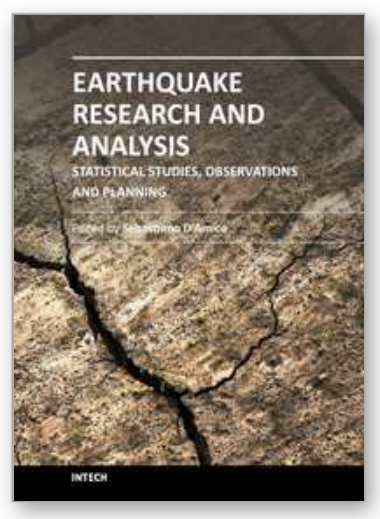

\author{
Earthquake Research and Analysis - Statistical Studies, \\ Observations and Planning \\ Edited by Dr Sebastiano D'Amico
}

ISBN 978-953-51-0134-5

Hard cover, 460 pages

Publisher InTech

Published online 02, March, 2012

Published in print edition March, 2012

The study of earthquakes plays a key role in order to minimize human and material losses when they inevitably occur. Chapters in this book will be devoted to various aspects of earthquake research and analysis. The different sections present in the book span from statistical seismology studies, the latest techniques and advances on earthquake precursors and forecasting, as well as, new methods for early detection, data acquisition and interpretation. The topics are tackled from theoretical advances to practical applications.

\title{
How to reference
}

In order to correctly reference this scholarly work, feel free to copy and paste the following:

Yasuichi Kitamura, Youngseok Lee, Ryo Sakiyama and Koji Okamura (2012). Experience with Restoration of Asia Pacific Network Failures from Taiwan Earthquake, Earthquake Research and Analysis - Statistical Studies, Observations and Planning, Dr Sebastiano D'Amico (Ed.), ISBN: 978-953-51-0134-5, InTech, Available from: http://www.intechopen.com/books/earthquake-research-and-analysis-statistical-studiesobservations-and-planning/experience-with-restoration-of-asia-pacific-network-failures-from-taiwanearthquake

\section{INTECH}

open science | open minds

\section{InTech Europe}

University Campus STeP Ri Slavka Krautzeka 83/A 51000 Rijeka, Croatia Phone: +385 (51) 770447 Fax: +385 (51) 686166 www.intechopen.com

\section{InTech China}

Unit 405, Office Block, Hotel Equatorial Shanghai No.65, Yan An Road (West), Shanghai, 200040, China 中国上海市延安西路65号上海国际贵都大饭店办公楼 405 单元 Phone: +86-21-62489820

Fax: $+86-21-62489821$ 
(C) 2012 The Author(s). Licensee IntechOpen. This is an open access article distributed under the terms of the Creative Commons Attribution 3.0 License, which permits unrestricted use, distribution, and reproduction in any medium, provided the original work is properly cited. 